Instability, Chaos and Predictability in Celestial Mechanics and Stellar Dynamics
Copyright 1992 by Nova Science Publishers, Inc. All rights of reproduction in any form reserved. ISBN 1-56072-054-9

\title{
STOCHASTICITY OF THE ORBIT OF THE ASTEROID 1989 AC
}

\author{
D. Benest, C. Froeschlé, and R. Gonczi \\ O.C.A. Observatoired de Nice, B.P. 139, F-06003 Nice Cedex, France
}

\section{ABSTRACT}

Using mainly Lyapunov Characteristic Indicators, the stochasticity of the orbit of the asteroid 1989 AC has been investigated in the framework of three-body, five-body and seven-body restricted models. From our first results, the orbit of 1989 AC has been found fairly chaotic, through an interplay of the $3 / 1$ resonance with Jupiter and close approaches with the inner planets.

\section{INTRODUCT ION}

Numerous extensive studies on dynamics of planet-crosser asteroids (for example by Milani et al., 1989) have shown a great diversity among the computed orbits; this diversity reflects evidently the chaotic nature of this kind of dynamics. Close approaches with the planets are now known to play a great role in the coming out of the chaos. Our aim here is to study the origin of the stochasticity for a recently discovered asteroid, 1989 AC (lately numbered and named 4179 Toutatis), nowadays near the $3 / 1$ resonance with Jupiter.

For this purpose, we compute the so-called Lyapunov Characteristic Indicators, quantities related to the now wellknown theory of Lyapunov Characteristic Exponents, which provide a quantitative measure of the stochasticity for a given orbit. For the numerical integration of the orbit, we have used first a seven-body model (Sun-Venus-Earth-Mars-AsteroidJupiter-Saturn). Then, in order to trace back the origin of the stochasticity, we have al so computed truncated planetary models: the first is the classical restricted three-body model 
(Gu-Jupiter-Asteroid), when the second model takes into account only the perturbations due to and thus the encounters with-the three Inner Planets cited above (Vems-Barth-Mars). Ve present here our first results.

\section{THE LYAPUNOV CHARACTERISTIC INDICATORS}

It is well known since the cla ssical study of Henon and Heiles ( 1964 ; see also Henon, 1981) that two or bits initially close diverge either linearly or exponentially depending on whether the initial points lie in an integrable or in a stochastic region of the phase space. This property has been extensivel y u sed as an indicator of stocha sticity, thr ough the socalled L yapunov Characteristic Exponents (hereafter called the ICE's); initially introduced by Lyapunov for periodic orbits, and further developed by 0 seledec, they indicate quantitatively how fast nearby or bits diverge and thus the degree of unpredictability of such orbits.

Generally, speaking, the LCE's are non uniform integrals of motion; therefore, if the dynamical system is ergodic,all the LCE's are almost everywhere constant; a contrario, if all the LCE's are not almost everywhere constant, then the dynamical system is certainly not ergodic. Practically, when we compute the ICE' $s$, if the maximal ICE tends to zero for $t \rightarrow \infty$, then the system is integrable; but if the maximal LCE tends to be constant for $t+\infty$ and a fortior $i$ if several other LCE's have the same behaviour), then the dynamical system is in the ergodic region (for more details, see Froeschle, 1984 , $1990)$.

We must keep in mind that the LCE's are stricto sensu $l$ imiting values at $t \rightarrow \infty$, which is evidently impossible to compute practicall $y$. We then def ine the Lyapunov Characteristic Indicators (hereafter called the ICI's) as the truncated values of the ICE's for a finite time $t y$. When the largest ICI ras a finite mn-zero value at $t_{M}$, we may consider the or bit as stoch stic with good confidence; but when it does not show any tendency to stop its monotonous decrea se down to zero, we only may suppose that it has a given probability tepending on $t_{M}$ ) to be integrable. Previaus computations have shown that a fairly good compramise betveen accuracy and reasorable computation time is $10^{4}$ years $<t_{y}<10^{5}$ years; nevertheless, when we suspect that a given or bit, a given orbit, althugh looking integrable at $t_{M}$, could be in fact chaotic, we can contine the computation to greater values of $t_{M}$.

\section{RESJLTS}

The a steroid $1989 \mathrm{AC}$ was discovered with the $0.9 \mathrm{~m}$ Scmidt Tele scope at Cau ssol s (Observatorire de la Cote d'Azur) Jamary the $4 \mathrm{th}$. The fast-moving object wa soon (I.A.U. Circular 4715, $1989 \mathrm{Jan} .13$ ) su spected to be at or near the $3 / 1$ resonance with Jupiter. For our study, we have taken as initial conditions the elements computed by Green (1989): 


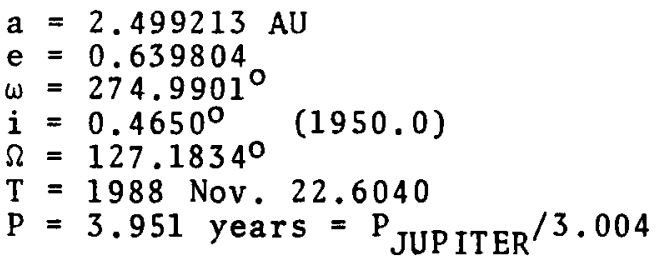

Elements of the planets at the initial time $T$ were taken from the Astronomical Ephemeris 1988 .

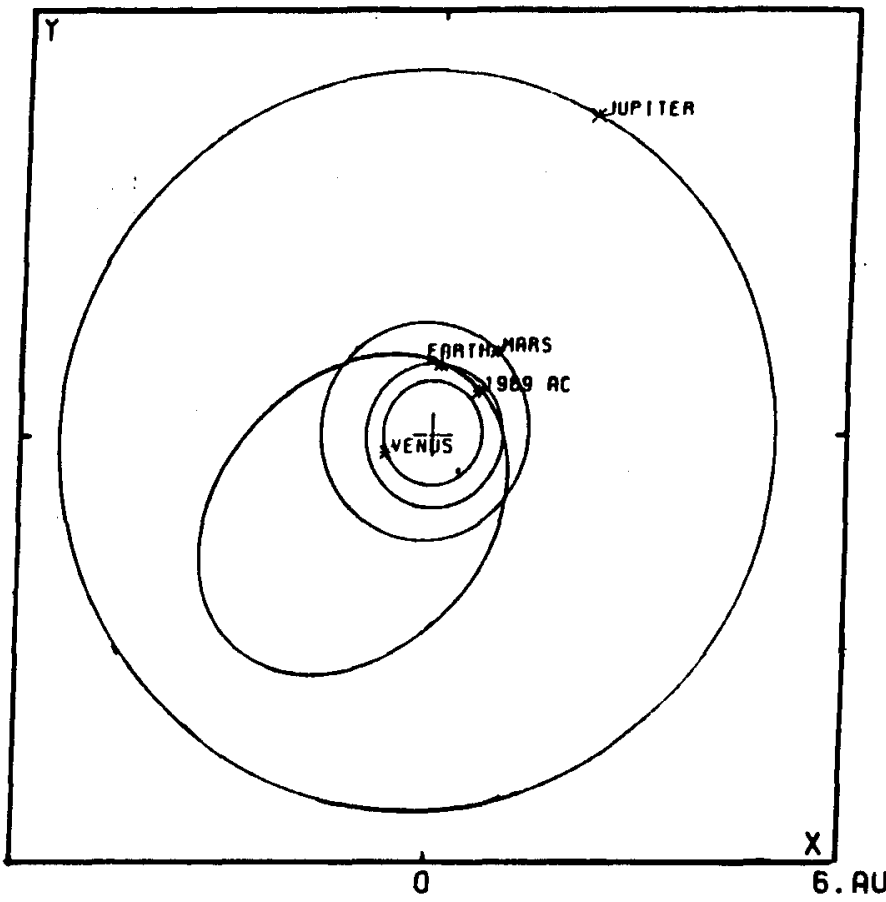

Fig. 1: Heliocentric orbit of Asteroid 1989 AC, together with Jupiter, Mars, Earth and Venus; the asterisks indicate the initial positions at epoch $\mathrm{T}=1988$ Nov. 22.6040 .

\subsection{The Sun-Jupiter-Saturn-Inner P1anets Model}

As the osculating orbit of $1989 \mathrm{AC}$ is located between the orbits of Venus and Jupiter (see Fig. 1), our main computation is made within the frame of a restricted seven body model, where the heliocentric orbit of $1989 \mathrm{AC}$ (with an intinitesimal mass) is perturbed by Venus, Earth, Mars, Jupiter and Saturn; this latter acts rather through its influence on Jupiter than directly on 1989 AC. All bodies are taken as point masses, submitted only to the newtonian gravitational 
force law. The equations of motion are integrated using the classical Bulirsch - Stoer method, which, through a controlled variable step size, allows to handle close approaches with planets much more accurately than with a fixed step size, as used for example by Milani et a1.(1989).

The evolution of the orbital elements and the LCI's is computed for 50000 years backward and 100000 years forward from the epoch $T$. The orbit appears to be strongly governed by the $3 / 1$ resonance with Jupiter, as the semi-major axis stays around 2.5 AU during a long time (see Fig. 2); but, due to repeated relatively close approaches with the inner pianets, the value of the eccentricity progressively increases, so that finally the asteroidal path plunges catastrophically inside the Sun. As regards the LCI's, the non-zero value of all of them indicates that the orbit is stochastic (see Fig. $3)$, and that no quasi-integral remains.

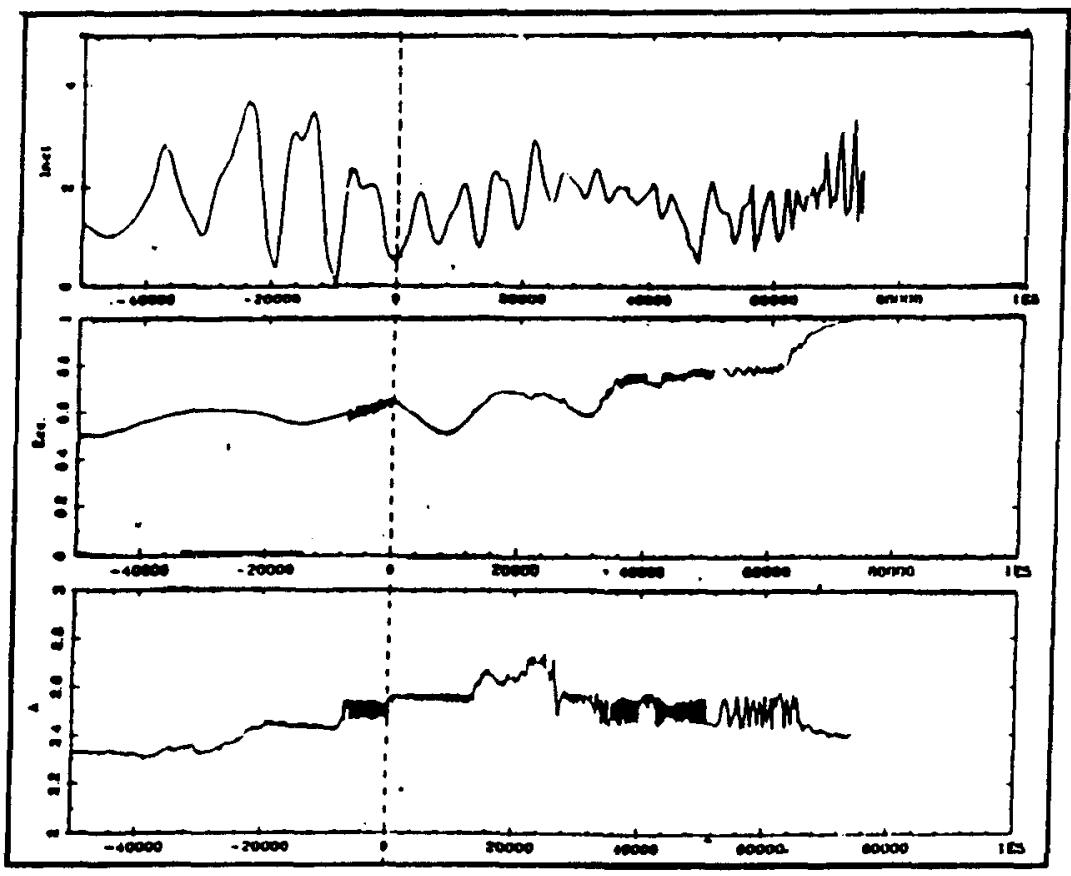

Fig. 2: Evolution of the semi-major axis $A$ and the eccentricity e of the "true" orbit of 1989 AC for 50000 years backward and 100000 years forward; seven-body model, Bulirsch-Stoer integrator; the vertical dashed 1 ine indicates the starting date $T$. 


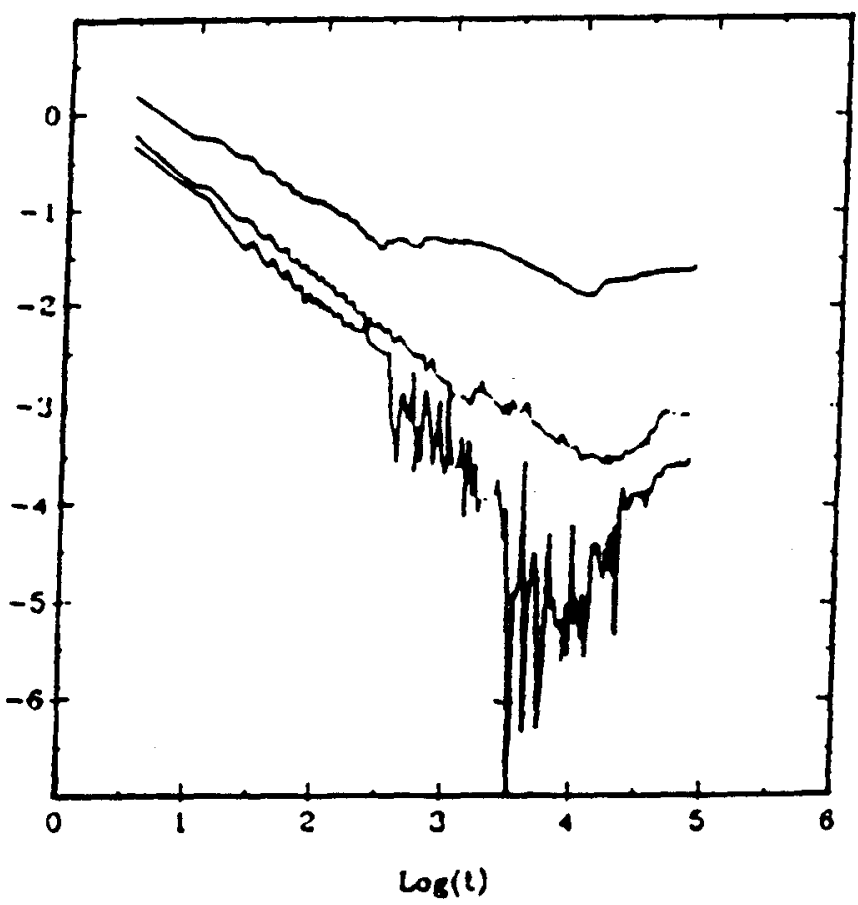

Fig. 3: Variation of the LCI's forward in time for the "true" orbit of 1989 AC; seven-body model, Bulirsch-Stoer integrator.

In this numerical experiment, we used the osculating elements of $1989 \mathrm{AC}$ at the epoch $\mathrm{T}$, with a zero initial value of the mean anomaly: $\mathrm{M}_{0}=0$. Then, another run was performed with the same seven-body model for an orbit initially very close to the former one (the "true" orbit): we changed only the initial value of the mean anomaly, by half a degree $M_{0}=$ $0.5^{\circ}$ ). This varied orbit stays in a first stage near the $3 / 1$ resonance with Jupiter, but suffers at around +50000 years a very strong encounter with the Earth, at a distance of $8 \times 10^{-4} \mathrm{AU}$; this encounter induces an increase of the semi-major axis, which allows later two very close encounters with Jupiter, at distances respectively of $6 \times 10^{-2} \mathrm{AU}$ and $3 \times 10^{-2} \mathrm{AU} ;$ a large jump in the semi-major axis upito great values occurs then, and the asteroidal orbit becomes an escape trajectory (see Fig. 4). 


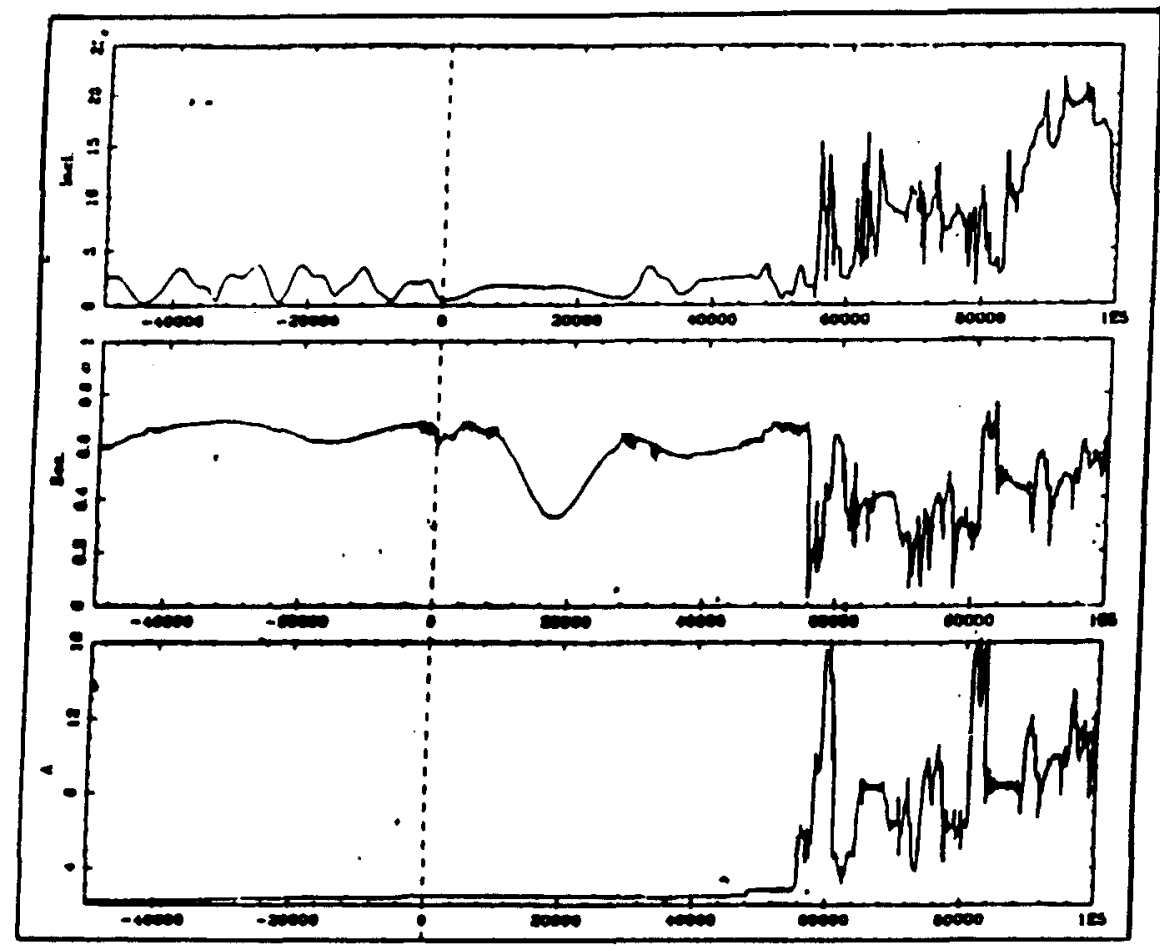

Fig. 4: Evolution of the semi-major axis $A$ and the eccentricity e of the varied orbit of $1989 \mathrm{AC}$; same notations as Fig. 2 .

Therefore, the "true" and the varied orbits, a1 though initially very close, diverge relatively rapidly and have very different final destiny; this is the charaxteristic of a chaotic behaviour, and it not astonishing that the LCI's of the "true" orbit (see Fig. 3) as well as the LCI's of the varied orbit (see Fig. 5) indicate this stochasticity. Nevertheless, the LCI's have the same values for the two orbits, which confirms their validity as quantitative indicators of the stochasticity.

On the other hand, the different orbital evolutions of these two initially close orbits show that the motion of $1989 \mathrm{AC}$ is under the combined influences of the $3 / 1$ resonance with Jupiter and the close approaches and encounters with the inner planets, which give the chaotic character cited above. In the following two sub-sections, 3.2 and 3.3 , we thus study separately the influence of the major planet Jupiter, and the influences of the inner planets, namely Venus, Earth and Mars. 


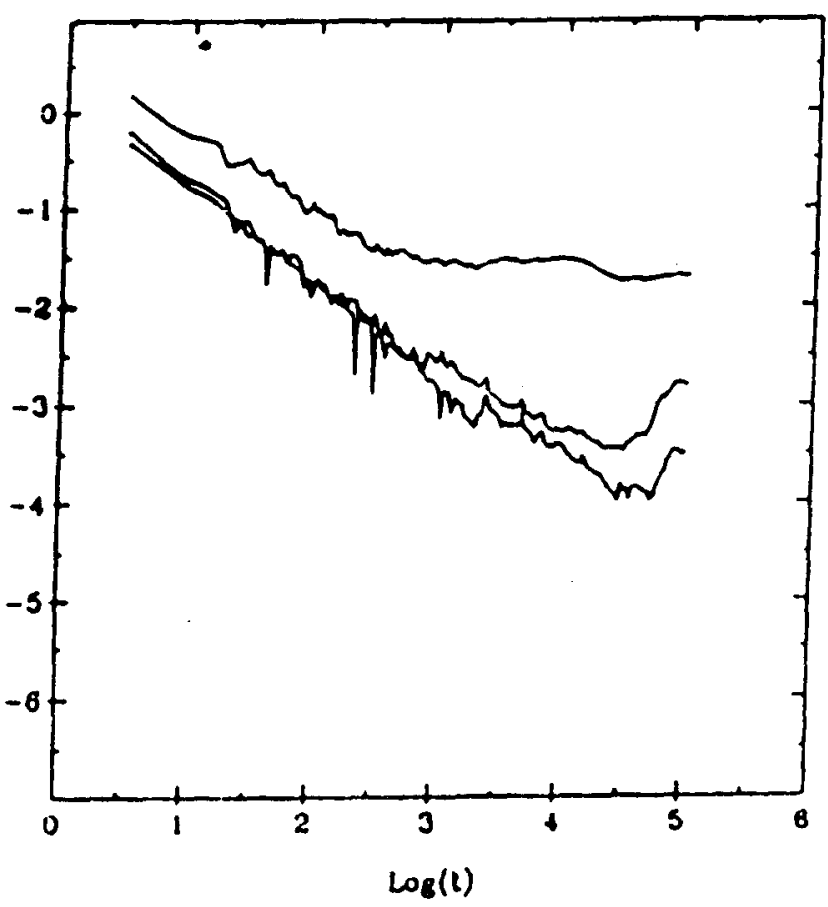

Fig. 5: Variation of the LCI's forward in time for the varied orbit of $1989 \mathrm{AC}$; same notations as for Fig. 3 .

\subsection{The Sun-Jupiter model}

The evolution of the orbital elements of $1989 \mathrm{AC}, \mathrm{compu}-$ ted for 200000 years backward and forward using a restricted three-body model (Sun-Jupiter-Asteroid) with the same integration technique as for the seven-body model, shows that the asteroid is locked in the $3 / 1$ resonance with Jupiter, at least during the considered interval of time (see Fig. 6). The LCI's do not show any non-zero limit (see Fig.7), as expected for such a near-integrable case. Therefore, Jupiter alone is not responsible for any tendency to stochasticity within the time span of 400000 years.

\subsection{The Inner Planets model}

In order to track the origin of stochasticity, we have performed computation using a restricted five-body model (SunVenus-Earth-Mars-Asteroid) with the same integration technique as above. Results (see Figs, 8 and 9) show a fair stochasticity: the largest LCI is less than the one with the seven-body integration, and the two others do not show any tendency to non-zero limit (Fig. 9). Multiple close approaches with the inner planets thus appear to play a non-negligible role in the stochasticity of the orbit of $1989 \mathrm{AC}$. 


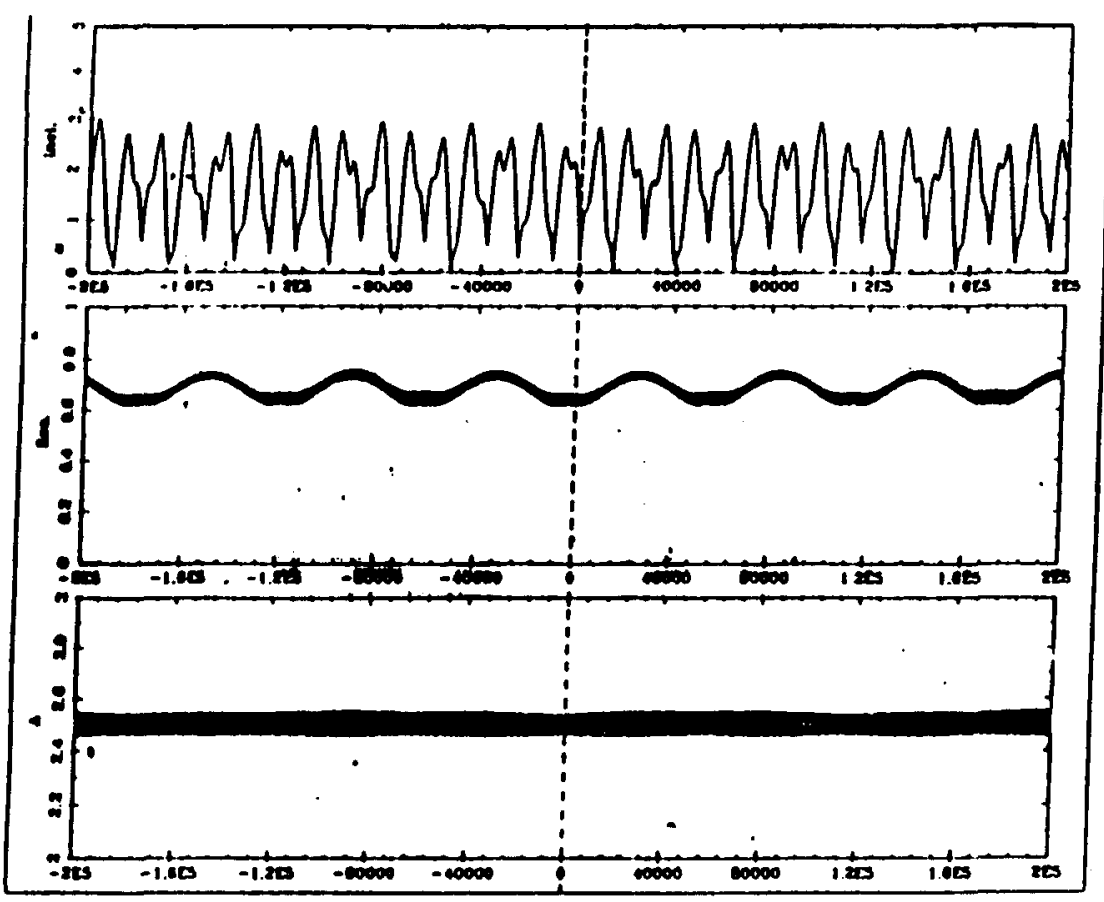

Fig. 6:Evolution of the semi-major axis $\Lambda$ and the eccentricity e of the orbit of 1989 AC for 200000 years backward and forward; three-body model; same notations as for Fig.2.

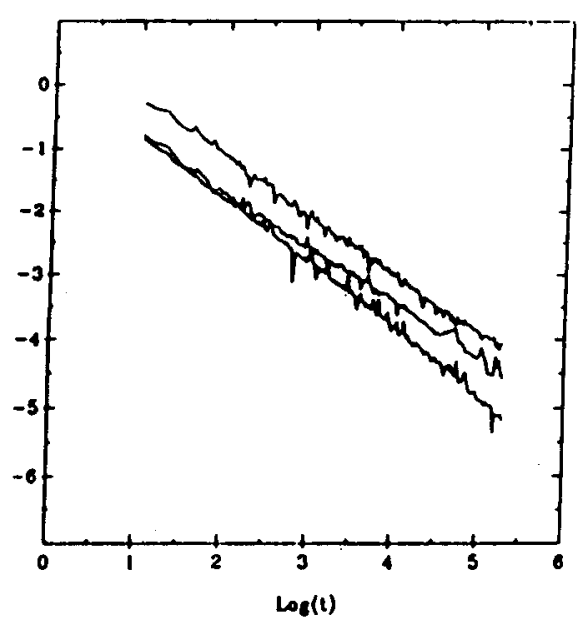

Fig.7: Variation of the LCI's forward in time for the orbit of $1989 \mathrm{AC}$; three-body model; same notations as for Fig. 3 . 


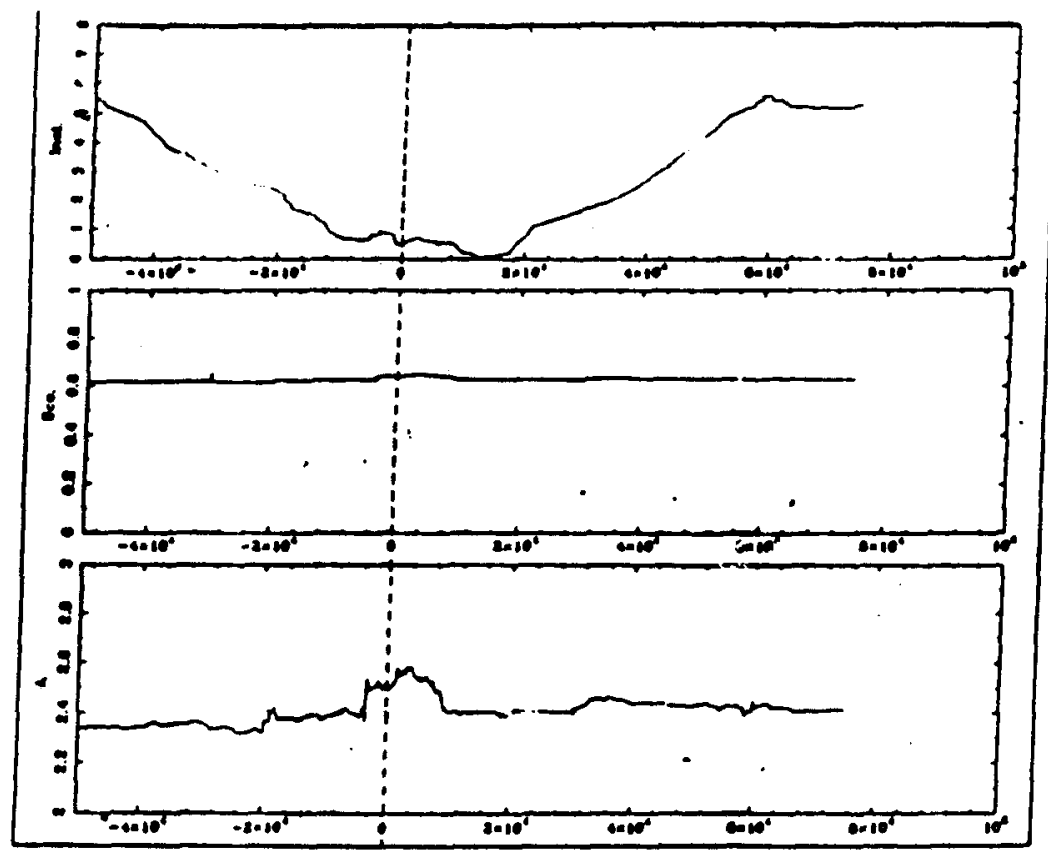

Fig.8: Evolution of the semi-major axis $A$ and the eccentricity e of the "true" orbit of 1989 AC for 50000 years backward and 100000 years forward;five-body model; same notations as for Fig. 2 .

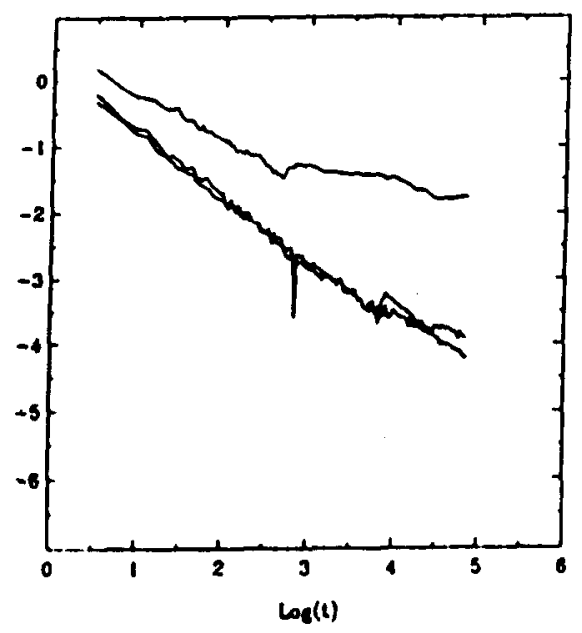

Fig.9: Variation of the LCI's forward in time for the orbit of $1989 \mathrm{AC}$; five-body model;same notations as for Fig.3. 


\section{CONCLUSION AND PERSPECTIVES}

Our first results indicate that the orbit of the asteroid $1989 \mathrm{AC}$ is chaotic. This stochasticity seems to be determined mainly by close approaches and encounters with the inner planets Venus, Earth and Mars. Nevertheless,Jupiter keeps some control on the evolution of the asteroid's orbit through the $3 / 1$ resonance.

We intend now to study the effects of the cares takes in the computation of close approaches by using different integration schemes, and through statistical analysis on the variations of the orbital elements.

\section{REFERENCES}

[1] C.Froeschle, The Lyapunov Characteristic Exponents Applications to Celestial Mechanisms,Celes Mech. 34,95$115(1984)$.

[2] C,Froeschié, Experiences Numeŕiques et Mécanique Céleste in Les Méthodes Modernes de la Mécanique Celeste,eds. D.Benest and C.Froesch16, Editions Frontieres,pp.331-367 (1990).

[3] D.W.E. Green, I.A.U. Circ. 4734 and M.P.C. 14204 (1989).

[4] M.Henon, Numerical Exploration of Hamiltonian Systems in Chaotic Behaviour of Deterministic Systems (Cours des Houches) 36,53-170(1981).

[5] M.Henon, C.Heiles, The Applicability of the third integral of Motion: Some Numerical Experiments, Astron.J.69, $73-79(1964)$.

[6] A.Milani, M.Carpino, G.Hahn, A.Nobili, Dynamics of Planet-crossing Asteroids: Classes of Orbital Behaviour, Icarus $78,212-269(1989)$. 